\title{
Prototype Design of a Ship Intelligent Integrated Platform
}

\author{
Fuxin $\mathrm{WANG}^{\mathrm{a}, 1}$, Hao $\mathrm{LUO}^{\mathrm{a}}$, Yuan $\mathrm{YU}^{\mathrm{b}}$ and Liyong $\mathrm{MA}^{\mathrm{b}}$ \\ a Automation Engineering Department, Shanghai Marine Diesel Engine Research \\ Institute, Shanghai, China \\ ${ }^{\mathrm{b}}$ School of Information Science and Engineering, Harbin Institute of Technology, \\ Weihai, China
}

\begin{abstract}
Recently, more and more attention has been paid to ship intelligence. However, in the sensor data acquisition network represented by the integrated ship bridge system, sensor data is collected and transmitted point to point, and the data coupling is strong, which is not conducive to the hierarchical utilization of data. To provide more effective data communication methods and flexible support to applications, intelligent integrated platform is needed by modern ship. Prototype design and practice of a ship intelligent platform is proposed, and the key technologies of the platform is discussed. The overall architecture of the platform is described. A hybrid network architecture with fieldbus, real-time ethernet and ethernet information network is introduced. And data storage architecture using NoSQL and hadoop distributed file system is described. The system can meet the real-time performance requirement of the control and information communication. An energy efficiency application based on the designed platform is developed, machine learning based method is employed to predict the heavy oil fuel consumption for ship navigation.
\end{abstract}

Keywords. intelligent ship, integrated platform, sensor, energy efficiency

\section{Introduction}

Recently, more and more attention has been paid to ship intelligence. The most typical traditional data collection system is the integrated ship bridge system [1]. The system collects sensor information centrally. And the functions of dispersed equipment such as navigation, driving control, collision avoidance, and surveillance are integrated into different systems with information fusion, which includes radar system, information system, and central driving control system. In this data collection method, the point-topoint communication method is used between the platform and shipboard, equipment, and subsystems. In this connection architecture, the coupling of the data is strong, and it brings inconvenience to the data update. It can be seen that modern ships need a smart application-oriented integrated intelligent platform design that can provide more effective data communication methods and more flexible support.

In recent years, some ships have carried out intelligent practice to change traditional data collection methods [2-5]. In 2015, South Korea's Hyundai Heavy Industry Group

\footnotetext{
${ }^{1}$ Corresponding author, Fuxin WANG, Automation Engineering Department, Shanghai Marine Diesel Engine Research Institute, Shanghai, China; E-mail: 18801909355@163.com.
} 
and Accenture jointly developed a new intelligent ship interconnection platform. The platform collects real-time dynamic information about the ship's position, equipment operation information, shipboard cargo information, and surrounding sea area information through sensors, and visually displays the analysis results through big data analysis. Danish Maersk selected the ShipManager system developed by DNVGL Group to collect and analyze information on its ships. GE Power Conversion has developed Visor Insight, a management system that can be applied to remotely monitor drive equipment, power distribution facilities, electric motors, and navigation systems.

Intelligent ships are also being rapidly developed in China. In 2015, the i-DOLPHIN ship was released by China State Shipbuilding Corporation, which means that China's first intelligent ship has started the design and construction. After that, China's intelligent ships flourished. For example, in 2017, the world's first intelligent ship "Dazhi" was officially delivered. This ship has the first ship smart operation and maintenance system with autonomous learning. In 2018, the 400,000-ton intelligent super-large ore ship "Mingyuan" was delivered to use. It is the world's largest intelligent ship with decisionmaking functions such as assisting collision avoidance. In 2019, the "Zhiteng" ship successfully carried out a demonstration of autonomous navigation and autonomous collision avoidance. The ship is equipped with an intelligent situational awareness system, autonomous navigation decision-making system, and autonomous driving control system. These are all necessary parts for ocean-going autonomous ships.

As more and more intelligent ships are built and used, research on intelligent ship integrated platforms is an urgent task. There are many sensors in intelligent ships, different types, and different communication methods. Big data and data monitoring applications for intelligent ship are proposed in [1,2], but the simple network cannot support complex intelligent applications. Navigation based on information fusion is discussed in [3-5], these applications do not consider the control data network. In this paper, network architecture considering both fieldbus and information network with high performance data storage method is introduced. With the development of data driven method [6-8], energy efficiency applications are focused by many researches $[9,10]$, and intelligent methods have been employed to energy efficiency applications. Neural network is the common method for energy consumption prediction $[11,12]$. In this paper an energy consumption prediction application based on machine learning is reported.

The main contribution of this paper is as follows. First, a ship integrated platform is designed and tested in Shanghai Marine Diesel Engine Research Institute. The key technologies of the intelligent integrated platform for ship is discussed. Second, a hybrid network architecture of fieldbus and real-time ethernet for sensor data collection is introduced, and hierarchical database architecture design is discussed. Third, an application for energy efficiency is implemented based on the integrated platform.

\section{Architecture of Intelligent Integrated Platform}

The China Classification Society officially released the Intelligent Ship Specifications on December 1, 2015, and the specifications have come into effect on March 1, 2016. This specification is the world's first intelligent ship specification and proposes to develop an intelligent integrated platform. In this specification, the intelligent integrated platform is one of the seven important components of intelligent ships. The intelligent integrated platform can realize data collection and unified storage, and it can also provide support for data analysis and intelligent applications. 
The overall architecture of an intelligent integrated platform is divided into seven parts, they are data acquisition, data storage, data integration, information application, information display, standard specification system, and information security system, as shown in Figure 1.

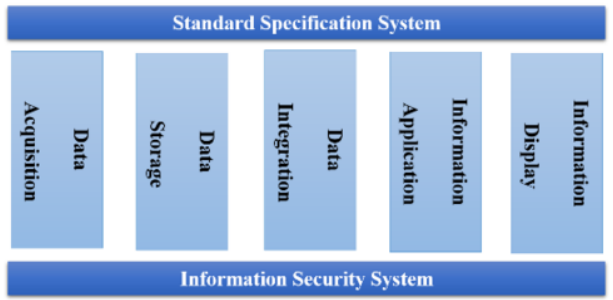

Figure 1. Architecture of intelligent integrated platform for ship.

In data acquisition, sensing devices, such as sensors, controllers, signal acquisition devices, and data acquisition devices, are used to collect the required data. In data storage, the dataset needs to be established to support the decision-making and management process, which is subject-oriented, integrated, relatively stable, and reflects historical changes. In data integration, the data is cleaned up and processed systematically, classified and summarized, analyzed, and integrated. In information applications, business applications are provided for customers. In information display, corresponding data mining results are provided human-computer interaction. The standard specification system and information security system are two other key requirements for data application. For the design and construction of a prototype platform, data acquisition, data storage, and data integration are the most important parts. These will be discussed in detail as follows.

\section{Network and Data Storage Design for Data Acquisition}

Due to the large number of ship information systems and the huge amount of information transmission between each other, the ship communication network has the characteristics of complexity and multi-channel, so the ship data communication network is the key problem for the design of an intelligent integrated platform. Ship communication must meet the needs of parallel multi-tasks. The main types of data transmission include audio, video, and other sensor data, the timeliness, and accuracy of data communication are also required.

In the design of the communication network, the method of separating the control layer and information layer is adopted. Because the control layer requires higher response time and synchronization time, CAN Fieldbus is applied. And the information layer does not require such high response time and synchronization time, real-time ethernet with better compatibility is adopted. EPA (Ethernet for plant automation) is a new open realtime ethernet standard for industrial field equipment, which is suitable for real-time communication of industrial measurement and control systems. EPA adds CSME between the data link layer and the network layer specified in ISO/IEC8802.3 protocol to control the transmission of real-time and non-real-time data packets, so as to ensure the timely transmission and processing of EPA periodic information and non-periodic information such as alarm. In addition, the EPA network divides the control network into 
several micro-network segments separated by a bridge in order to avoid the occurrence of conflict, and the communication in each micro-network segment does not interfere with each other. EPA based distributed network control system has been successfully applied in the factory. The real-time response time of EPA is less than 1 millisecond. EPA control module CON21 and ECS-100 are used in field control terminals and system center stations respectively. The control layer adopts distributed control, which is connected to dual redundant optical fiber Ethernet through a redundant gateway to realize the interconnection with the upper information management network. The network architecture is illustrated in Figure 2. As far as we know, this is the first integrated platform network design and practice for fieldbus, real-time ethernet and information ethernet, and its network real-time performance can reach 1 millisecond.

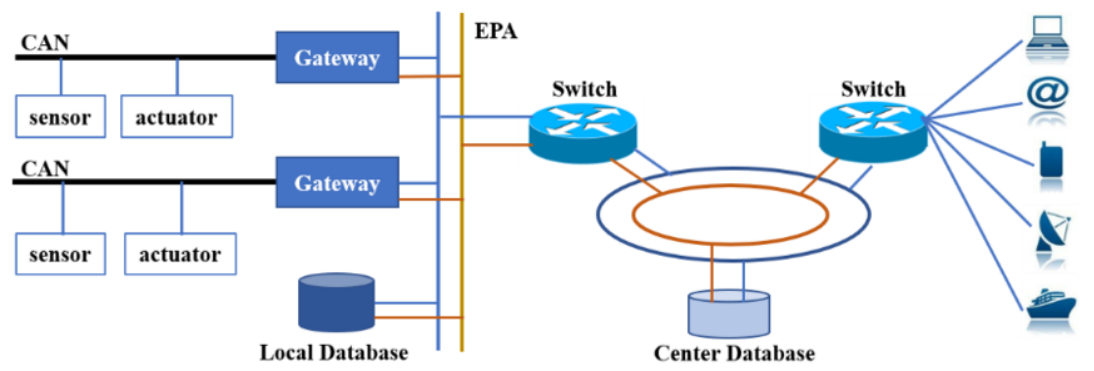

Figure 2. Network architecture for integrated platform.

The database is the key design of data storage. The real-time database used in the integrated platform consists of two parts: the field database and the central database. The on-site database is responsible for data acquisition, compressed storage, and processing to provide on-site monitoring data services. The central database is responsible for synchronous storage and processing of all field data to provides a data query interface. AutoNet, which is widely used in ship engineering, is used as the field real-time database system of the platform.

Different from the traditional solution to achieve high performance of the system through the high reliability of hardware, some NoSQL distributed databases can use lowcost servers to build large-scale clusters and provide unified external memory access services. In the current data explosion and cost considerations, more and more distributed database products are used to replace RDBMS. Some studies have compared the performance of some NoSQL and SQL databases in reading, write, delete and instantiation operations. Although for different databases, their performance will change with different operations, but in the case of large operands, NoSQL shows its advantages. As a typical representative of the distributed NoSQL database, HBase has been widely used in sensor data storage. HBase is a distributed NoSQL open source database, it has the characteristics of high availability, high performance, and scalability of the distributed storage system. HDFS (Hadoop distributed file system) is a kind of distributed file system, which is used to solve the storage problem of massive data. It can manage the files on multiple machines at the same time and realize the file sharing on multiple machines. It has strong fault tolerance and stability. And it can be combined with HBase to achieve efficient data file support. In the prototype design of the intelligent integrated platform in this paper, HBase is used as the central database and HDFS as the file system. The database in the platform is shown in Figure 3. This database architecture with the previous network architecture, can fully guarantee the real-time performance of 
data. Compared with the schemes in the literature [1-3], this scheme not only considers the hybrid network architecture, but also considers the database architecture. The scheme ensures the real-time performance of the integrated platform more completely.

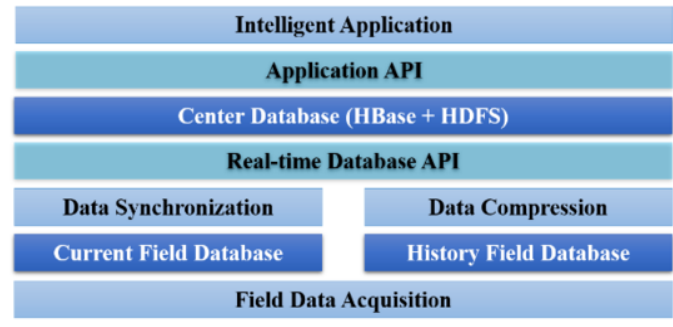

Figure 3. Database in the integrated platform.

\section{Application of Ship Energy Efficiency with Data Integrated Platform}

The purpose of ship intelligent integrated platform is to provide a unified data interface and support for data analysis in different subsystems. The value of these integrated data can only be fully exploited by using statistics, machine learning, and other artificial intelligence technologies. Various data analysis applications are the further expansion and extension of the data in the intelligent integrated platform. With the emergence of emerging technologies such as big data and artificial intelligence, as well as the continuous maturity of data mining technology, these data analysis applications will bring more opportunities and challenges. These applications can extract hidden and potentially useful information and knowledge from a large number of practical application data. This is the characteristic and construction purpose of an intelligent platform. The ship's intelligent integrated platform provides strong support for these applications.

Using the intelligent integrated platform, some preliminary applications have been developed by our team. In this paper, we introduce the application of energy efficiency. Energy-saving has always been an important goal in ship design and operation management [6]. A basic ship navigation energy consumption model is established, which is based on the marine environment, ship navigation, and energy consumption data. The marine environment includes wind speed, wind direction, air temperature, air pressure, and sea wave height. These data come from the marine environment sensing module of the ship, which is obtained through its measurement or shore-based communication. The ship navigation module can provide the ship's actual speed and heading data information. The diesel engine real-time monitoring module can provide speed and diesel consumption data. Although these data come from different subsystems, a unified interface can be used to access and query historical data through the integrated platform.

When the diesel engine working condition and the ship's heading are stable, the data within 20 minutes is collected and preprocessed. Finally, the shipping speed, ship heading course, wind speed, wind direction, air temperature, air pressure, wave height, and diesel consumption are used for data analysis. All this data comes from a newly constructed cargo ship undergoing sea trials. A total of 328 pieces of valid data were collected, of which 295 were used as training dataset for model building, and the remaining 33 were used as test dataset for model testing. 
The support vector machine (SVM) is a classifier developed with the statistical learning theory. The feature of SVM is that a largest separation hyperplane is found for classification. The hyperplane is established by maximizing the interval between the two classes in the feature space. This problem can be solved with optimization algorithm. The support vector machine method based on statistical learning theory can also be used to deal with regression problems, this method is called support vector regression (SVR). In our application based on the integrated platform, SVR is used to estimate heavy oil fuel consumption. At first, a model is established with the training dataset employing SVR. Then the trained model is used to estimate the heavy oil fuel consumption according to other known parameters. The experimental results show that the maximum relative error is $9.11 \%$, the average relative error is $3.35 \%$, the maximum absolute error is $3.5 \mathrm{~kg} / \mathrm{km}$, and the average absolute error is $1.4 \mathrm{~kg} / \mathrm{km}$. The results are shown in Figure 4. This shows that the model can be used to predict fuel consumption. And the performance of this method is similar to that reported in other literatures [9-12]. Moreover, the model can be used to select the best ship speed based on energy efficiency under known navigation conditions. More accuracy prediction model can be established when more data are collected or some other intelligent algorithms are employed.

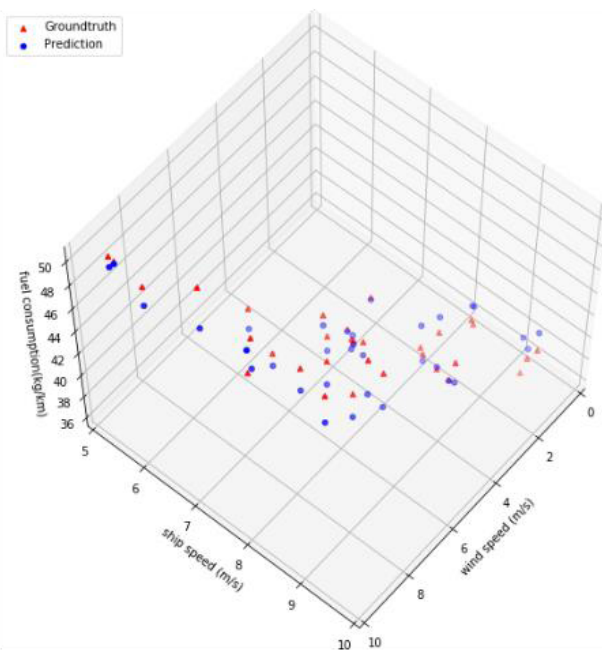

Figure 4. Prediction results of fuel consumption.

\section{Conclusions}

Prototype design and practice of a ship intelligent platform is proposed. The network architecture and data storage architecture are the key designs of the platform. A hybrid network architecture of fieldbus and real-time ethernet for sensor data is used, and NoSQL database and hadoop distributed file system is employed to obtain real-time performance. An energy efficiency model is established with a machine learning method, and it can be used to predict fuel consumption. The example shows the supporting role of the platform for intelligent applications.

The limitations of this study mainly include two aspects. First, more data is needed to test the network performance. Second, more intelligent applications are needed to 
verify the rationality of the platform design. We will apply the platform design to more ships in the future. And more network and database testing will be done. At the same time, more intelligent applications based on the integrated platform will be developed.

\section{References}

[1] Li G, Deng X, Zhou M, et al. Research on data monitoring system for intelligent ship. Lecture Notes in Electrical Engineering. 2020 Nov;634(1):234-241.

[2] Jia S, Ma L, Zhang S. Big data prototype practice for unmanned surface vehicle. In: Proceedings of the 4th International Conference on Communication and Information Processing; 2018 Nov 2-4; Qingdao, China: ACM Press; p. 43-47.

[3] Zhang C, Guo C, Guo MZ. Information fusion based on artificial intelligence method for SINS/GPS integrated navigation of marine vessel. Journal of Electrical Engineering and Technology. 2020 May;15(3):1345-1356.

[4] Xu K. A self-selective correlation ship tracking method for smart ocean systems. Sensors. 2019 Feb;19(4):8211-8219.

[5] Ma L, Xie W, Huang H. Convolutional neural network based obstacle detection for unmanned surface vehicle. Mathematical Biosciences and Engineering. 2020 Jan;17(1):845-861.

[6] Malde K, Handegard NO, Eikvil L, Salberg A. Machine intelligence and the data-driven future of marine science. ICES Journal of Marine Science. 2019 Jul;77(4): 1274-1285.

[7] Prera LP, Mo BS. Machine intelligence based data handling framework for ship energy efficiency. IEEE Transactions on Vehicular Technology. 2017 Oct;66(10):8658-8666.

[8] Park JH, Storch RL. Overview of ship-design expert systems. Expert Systems. 2002 Jul;19(3):136-141.

[9] Bharati S, Podder P, Mondal M. Visualization and prediction of energy consumption in smart homes. International Journal of Hybrid Intelligent Systems. 2020 Feb;16(2):81-97.

[10] Bharati S, Rahman MA, Mondal R, Podder P, et al. Prediction of energy consumed by home appliances with the visualization of plot analysis applying different classification algorithm. Advances in Intelligent System and Computing. 2020 Nov;1014(1):246-257.

[11] Farag YB, Ölçer AI. The development of a ship performance model in varying operating conditions based on ANN and regression techniques. Ocean Engineering. 2020 Feb;198(1): Article No. 106972.

[12] Le LT, Lee G, Park KS, Kim H. Neural network-based fuel consumption estimation for container ships. Korea. Maritime Policy \& Management. 2020 Jul;47(5): 615-632. 